\title{
Non-Isothermal Crystallization Kinetics of Poly(ethylene terephthalate) from the Point of View of Isokinetic Models
}

\author{
Markus LAMBRIGGER \\ CRPP-Technologie de la Fusion, Ecole Polytechnique Fédérale de Lausanne, \\ CH-5232 Villigen PSI, Switzerland
}

(Received August 7, 1997)

\begin{abstract}
KEY WORDS Differential Scanning Calorimetry / Inflexion Point / Nakamura Equation / Polymer Crystallization / Kinetics /
\end{abstract}

The kinetic crystallization model of Avrami is the generally accepted starting point for the analysis of isothermal nucleation and crystallization of polymers. ${ }^{1-3}$ It will be shown in this communication that, in the case of non-isothermal crystallization experiments performed with constant heating or cooling rates and analyzed by using differential scanning calorimetry (DSC), even an apparent $m$-order reaction model, being defined for every real, positive reaction-order $m$, is appropriate to describe the corresponding crystallization curves in the vicinity of the inflexion points. The latter approach will be shown to be approximately equivalent to the isokinetic nucleation and growth model of Nakamura ${ }^{4}$ as well as to the non-isothermal crystallization kinetics theory applied to DSC-curves of Ozawa. ${ }^{5}$ The apparent $m$-order reaction model is given by the following equation, ${ }^{6}$ if constant heating or cooling rates are maintained during the DSC-experiments:

$$
\begin{gathered}
\frac{\partial \alpha(T)}{\partial T}=\frac{K_{0}}{q} \exp \left(\frac{-E_{\mathrm{R}}}{R T}\right)(1-\alpha(T))^{m} \\
k_{\alpha}(T)=K_{0} \exp \left(\frac{-E_{\mathrm{R}}}{R T}\right) \\
\frac{\partial k_{\alpha}(T)}{\partial T}=k_{\alpha}(T)\left(\frac{E_{\mathrm{R}}}{R T^{2}}\right)
\end{gathered}
$$

whereby $\alpha(T)$ represents the conversion factor at the absolute temperature $T$ (varying from 0 to 1 ), $K_{0}$ the frequency factor, $R$ the gas constant, $E_{\mathrm{R}}$ the activation energy, $q$ the heating or cooling rate, $k_{\alpha}(T)$ the temperature-dependent rate constant and $m$ the apparent reaction-order. The inflexion point of the function $\alpha(T)$ is denoted by $\alpha\left(T_{\mathrm{in}}\right)$. It is found by solving the equation

$$
\left.\frac{\partial^{2} \alpha(T)}{\partial T^{2}}\right|_{T=T_{\mathrm{in}}}=0
$$

This has already been done in a previous publication. ${ }^{6}$ The following result has been obtained:

$$
\alpha\left(T_{\text {in }}\right) \approx 1-m^{1 /(1-m)}
$$

In deriving eq 5 some approaches have been made assuming that the investigated chemical reactions, curing reactions, phase transformations or crystallization processes occur over a relatively narrow temperature range around $T_{\text {in }}$ in comparison with the absolute temperature $T$. This assumption is justified in most practical cases. Thus, $\alpha\left(T_{\text {in }}\right)$ has been found to be approximately independent of the temperature, the temperature-dependent rate constant and the heating (or cooling) rate $q . \alpha\left(T_{\text {in }}\right)$ is only dependent on the apparent reaction-order $m$. Because $m$ is approximately independent of $q$, if an inflexion point exists, eq 5 is valid for a wide range of $q$-values, although not for $q=0 .{ }^{6} \mathrm{On}$ the other side, the isothermal crystallization model of Avrami, being based on the nucleation and growth characteristics of crystalline phases, also shows an inflexion point for $n>1$ as well as for the formal cases with $n<0$. The three-parameter Avrami equation function, ${ }^{7,8}$ being the result of the isothermal nucleation and growth theory, is given by

$$
\theta(t)=1-\exp \left[-k(t-\tau)^{n}\right]
$$

whereby $\theta(t)$ is the relative crystallinity at time $t, k$ the crystallization rate constant containing the nucleation and growth rates, $\tau$ the induction period and $n$ the Avrami index. Moreover, the value of $\theta\left(t_{\text {in }}\right)$ at the inflexion point is given by ${ }^{7}$

$$
\theta\left(t_{\text {in }}\right)=1-\exp \left[\frac{(1-n)}{n}\right]
$$

If $\alpha\left(T_{\text {in }}\right)$ is formally set equal to $\theta\left(t_{\text {in }}\right)$, the following relation between the Avrami index $n$ and the apparent reaction-order $m$ is found ${ }^{6}$ :

$$
n \approx \frac{1}{\ln \left[m^{1 /(1-m)}\right]+1}
$$

Equation 8 implies that, with the exception of the singularity at $m=1$, every real, positive value of an apparent reaction-order $m$ corresponds exactly to one real value of $n$, being larger than one or smaller than zero. ${ }^{6}$ Thus, Avrami's microscopic, isothermal crystallization model of nucleation and growth is on the macroscopic scale, with respect to the crystallization process in the vicinity of the inflexion point, nearly equivalent to an apparent, non-isothermal $m$-order reaction model; however, the formal reaction-order $m$ must be defined for every positive, real value. ${ }^{6}$ Thus, the reaction-order $m$ can also be considered as an atomic parameter, which determines the dimensionality of the growing solid phase-domains of any chemical reaction during the time-period characterized by the maximum 
reaction rate. Besides, the inflexion point region of the Avramic curve denotes the period of maximum reaction rate. Moreover, according to eq $8, m$ is related to the dimensionality of the growing domains in a highly non-linear manner.

On the basis of isokinetic conditions (identical timedependence of nucleation rate and growth rate), the neglection of induction periods and the assumption that the number of activated nuclei is constant, Nakamura et $a l{ }^{4}$ developed the following equation from the Avrami theory ${ }^{1-3}$ :

$$
\theta(t)=1-\exp \left[-\left(\int_{0}^{t} K(T) \mathrm{d} \tau\right)^{n}\right]
$$

In the case of a constant cooling or heating rate $q$, eq 9 can be reformulated as a function of $T$ :

$$
\theta(T)=1-\exp \left[-\frac{1}{q^{n}}\left(\int_{0}^{T} K(T) \mathrm{d} T\right)^{n}\right]
$$

whereby $n$ represents the Avrami index obtained from isothermal crystallization experiments, and $K(T)$ denotes the non-isothermal crystallization rate constant. $K(T)$ is related to the isothermal crystallization rate constant $k(T)$ by $^{4}$

$$
K(T)=[k(T)]^{1 / n}
$$

Differentiation and rearrangement of eq 9 provide the differential form of the isokinetic Nakamura equation ${ }^{9}$ :

$$
\frac{\partial \theta(t)}{\partial t}=n K(T)(1-\theta)[-\ln (1-\theta)]^{(n-1) / n}
$$

In the case of constant cooling or heating rates $q$, eq 12 can be reformulated as follows:

$$
\frac{\partial \theta(T)}{\partial T}=\frac{n}{q} K(T)(1-\theta)[-\ln (1-\theta)]^{(n-1) / n}
$$

Another expansion of the Avrami theory to nonisothermal experiments, being based on the assumption of a Poisson probability distribution of the crystallization events, but neglecting induction periods and being restricted to experiments with constant heating or cooling rates, has been suggested by Ozawa. ${ }^{5}$ The non-isothermal Ozawa equation function is given by

$$
\theta(T)=1-\exp \left[\frac{-\chi(T)}{q^{n}}\right]
$$

whereby $\chi(T)$ represents the heating or cooling function. Besides, the neglection of the induction periods $\tau$ is usually not a critical point, if merely non-isothermal crystallization experiments are studied. In the case of constant cooling or heating rates, there exists the following relation between the Ozawa and Nakamura equations:

$$
\chi(T)=\left[\int_{0}^{T} K(T) \mathrm{d} T\right]^{n}
$$

If the modified Nakamura eq 13 is applied and if it is assumed that the absolute value of the first derivative $|\partial k / \partial T|$ of the isothermal crystallization rate constant $k$, being related to eq 6 , is merely of the same magnitude in the vicinity of the isothermal inflexion point like $\partial k_{\alpha} / \partial T$ given by eq 3 , eq 7 and 8 may also be applied to the case of non-isothermal crystallization experiments performed with constant heating or cooling rates $q$. Moreover, since $k$ is also a kind of reaction rate like $k_{\alpha}$ in the case of cold-crystallization, and since the value of the first derivative of the isothermal crystallization rate constant $\partial k / \partial T$ is, in the case of melt-crystallization, approximately equivalent to the negative value of the first derivative of the isothermal crystallization rate constant $k$ received by performing cold-crystallization experiments with the corresponding $q$-rates, ${ }^{6}$ the assumption stated above is reasonable at least for experimentally relevant values of $n .^{10,11}$ Thus, every continuous, non-isothermal crystallization curve $\theta(T)$, obtained by applying a constant rate $q$ and showing one significant inflexion point with a distinct slope $\partial \theta(T) /$ $\left.\partial T\right|_{T=T_{\text {in }}}$, can be approached in the vicinity of the inflexion points by an apparent $m$-order reaction function of the type given by eq 1 , whereby $m$ is calculated by eq 7 and 8 , and the temperature-dependent rate constant $k_{\alpha}$ is obtained by fitting $\partial \alpha(T) /\left.\partial T\right|_{T=T_{\text {in }}}$ to the experimental slope $\partial \theta(T) /\left.\partial T\right|_{T=T_{\text {in }}}$. Since $\theta\left(t_{\text {in }}\right)$ is approximately independent of $q, \theta\left(t_{\mathrm{in}}\right)$ can be simply set equal to $\theta\left(T_{\text {in }}\right)$. Therefore, Avrami's isothermal ${ }^{1-3}$ model and Nakamura's and Ozawa's non-isothermal crystallization models of nucleation and growth ${ }^{4,5}$ are, on the macroscopic scale and with respect to the crystallization process in the vicinity of the inflexion point, nearly equivalent to the non-isothermal $m$-order reaction model and vice versa. However, this equivalence requires that the formal reaction-order $m$ is defined for any positive, real value, and that the corresponding Avrami index $n$ is larger than one or smaller than zero. As a matter of fact, $n<0$ in the Avrami equation characterizes a kind of dissolution process of a fully crystallized material, whereas $n>0$ defines the dimensionality of the growing crystallites starting from a melt or a glassy state.

If experiments with constant cooling or heating rates are considered, eq 7 and 13 can be combined providing an expression for $K\left(T_{\text {in }}\right)$ :

$$
\begin{aligned}
& K\left(T_{\text {in }}\right) \\
& \quad=\left.\frac{q}{n}\left[\left(\frac{n-1}{n}\right)^{(n-1) / n} \exp \left(\frac{1-n}{n}\right)\right]^{-1} \frac{\partial \theta(T)}{\partial T}\right|_{T=T_{\text {in }}}
\end{aligned}
$$

Furthermore, a comparison of eq 7 and 9 provides:

$$
\int_{0}^{t_{\text {in }}} K(T) \mathrm{d} \tau=\left(\frac{n-1}{n}\right)^{1 / n}
$$

Finally, the combination of eq 7, 14, and 15 leads to the heating (or cooling) function of the Ozawa equation at the inflexion point:

$$
\chi\left(T_{\mathrm{in}}\right)=\left(\int_{0}^{T_{\mathrm{in}}} K(T) \mathrm{d} T\right)^{n}=\left(\frac{n-1}{n}\right) q^{n}
$$

By performing DSC-measurements with varying $q$, the temperature $T_{\text {in }}$ can also be changed considerably thus providing the basis for the determination of the temperature dependent functions $K(T)$ and $\chi(T)$. Chan 
Table I. DSC-data obtained by non-isothermal cold-crystallization of PET $^{9}$ and evaluated by the inflexion point method

\begin{tabular}{|c|c|c|c|c|c|c|c|c|}
\hline $\begin{array}{l}\text { Scan } \\
\text { rate } q\end{array}$ & & $T_{\mathrm{in}}$ & $\frac{\partial \theta\left(T_{\mathrm{in}}\right)}{\partial T}$ & & & $K\left(T_{\text {in }}\right)$ & $k\left(T_{\text {in }}\right)$ & $\chi\left(T_{\text {in }}\right)$ \\
\hline$\left(\frac{{ }^{\circ} \mathrm{C}}{\mathrm{s}}\right)$ & & ${ }^{\circ} \mathrm{C}$ & $\left(\frac{1}{{ }^{1} \mathrm{C}}\right)$ & index $n$ & $\begin{array}{l}\text { reaction-order } \\
m\end{array}$ & $\left(\frac{1}{s}\right)$ & $\left(\frac{1}{s^{n}}\right)$ & $\left(\frac{{ }^{\circ} \mathrm{C}^{n}}{\mathrm{~s}^{n}}\right)$ \\
\hline 0.042 & 0.45 & 131 & 0.079 & 2.4 & 2.65 & $3.34 \times 10^{-3}$ & $1.14 \times 10^{-} 6$ & $2.90 \times 10^{-4}$ \\
\hline 0.083 & 0.45 & 138 & 0.093 & 2.4 & 2.65 & $7.89 \times 10^{-3}$ & $8.98 \times 10^{-6}$ & $1.48 \times 10^{-3}$ \\
\hline 0.167 & 0.45 & 148 & 0.112 & 2.4 & 2.65 & $1.91 \times 10^{-2}$ & $7.51 \times 10^{-5}$ & $7.95 \times 10^{-3}$ \\
\hline 0.333 & 0.45 & 162 & 0.168 & 2.4 & 2.65 & $5.72 \times 10^{-2}$ & $1.04 \times 10^{-3}$ & $4.17 \times 10^{-2}$ \\
\hline
\end{tabular}

Table II. DSC-data obtained by non-isothermal melt-crystallization of $\mathrm{PET}^{9}$ and evaluated by the inflexion point method

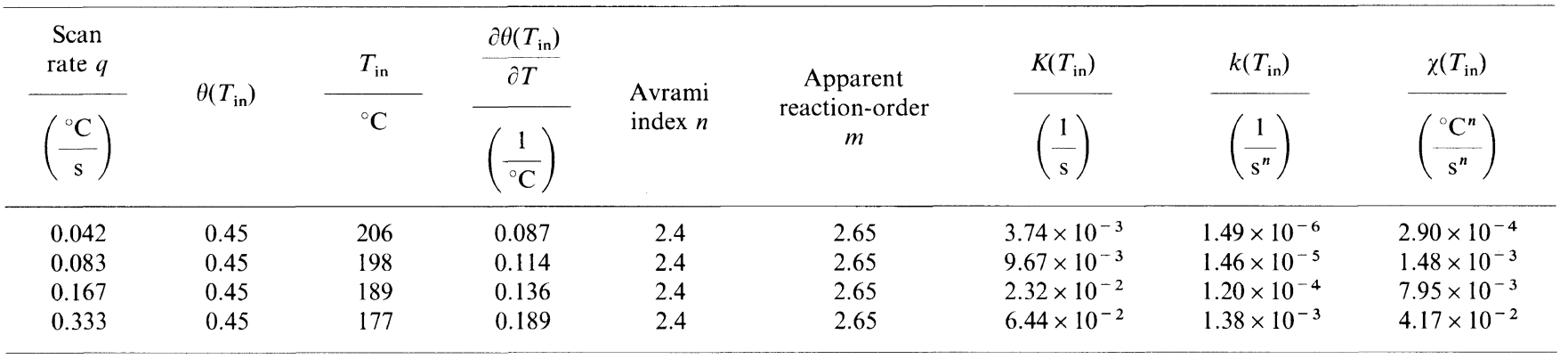

and Isayev $^{9}$ have reported non-isothermal cold- and melt-crystallization data of Polyethylene Terephthalate (PET) 7352 resin manufactured by Eastman Chemical Company. DSC-experiments with constant heating and cooling rates $q$ have been performed. Their data have been analyzed graphically and with the help of eq 7, 8, 11,16 , and 18 using the inflexion point method presented above. The results of these evaluations are tabulated in Tables I and II. It is observed that $\theta\left(T_{\text {in }}\right)$ is only dependent on $n$, i.e., independent of heating and cooling rates $q$, as it is expected following the theory presented in the previous sections. Moreover, every experimental, nonisothermal crystallization curve $\theta(T)$ shows only one inflexion point independent of the cooling or heating rate $q,{ }^{9}$ facilitating the application of the theory based on apparent $m$-order reaction functions of the type given by eq 1 . The highest precision in determining the Avrami index $n$ graphically is achieved for small slopes $\partial \theta(T) /\left.\partial T\right|_{T=T_{\text {in }}}$, i.e., a low $T_{\text {in }}$ for melt crystallization and a high $T_{\text {in }}$ for cold-crystallization. Small slopes are technically accomplished by performing DSC-measurements with relatively high cooling or heating rates $q .{ }^{9}$ The $n$-values, which have been evaluated graphically with the inflexion-point method, are equal for cold- and melt-crystallization $(n \approx 2.4) .{ }^{6,9}$ The isokinetic approach of Nakamura et al. ${ }^{4}$ is experimentally clearly supported by the results displayed in Tables I and II, since a approximately symmetrical distribution of $k\left(T_{\text {in }}\right)$ is obtained for cold- resp. melt-crystallization with respect to the temperature-mark at about $170^{\circ} \mathrm{C} .{ }^{6}$ Moreover, the results for $k\left(T_{\text {in }}\right)$ and $\chi\left(T_{\text {in }}\right)$ are in qualitative agreement with data reported elsewhere for isothermal and non-isothermal melt-crystallization of PET. ${ }^{12,13}$

It is concluded that the kinetics of polymer crystallizations, being characterized by a single Avrami index $n$, can be described in the vicinity of the inflexion point as well with a nucleation and crystal-growth model as, formally, with an apparent $m$-order reaction model. However, this is only possible, if the corresponding Avrami index $n$ is larger than one or smaller than zero. On the other side, any type of chemical process, whose kinetics can be described by an apparent $m$-order reaction equation of the type given by eq 1 , can also be interpreted in terms of the Nakamura ${ }^{4}$ or Ozawa ${ }^{5}$ theory in the vicinity of the inflexion points. However, the crystallites might, sometimes, be imaginary belonging to virtual spaces of dimensions $d>3$ or $d<0$, depending on the value of the Avrami index $n .^{5}$ The Avrami index $n$ is non-ambiguously related to the dimension of the growing crystallites. Nevertheless, the exact relation is also influenced by the type of growth control (diffusion- or interface-controlled) and by the type of nucleation (instantaneous nucleation or constant nucleation rate), ${ }^{5,14}$ but, in any case, $d-1.5 \leq n \leq d+1$ is valid. ${ }^{14}$ Thus, according to eq $8, d>3$ can result easily for apparent reaction orders $1<m<\approx 2$, whereas $d<0$ are possible values for $0<m<1$.

\section{REFERENCES}

1. M. Avrami, J. Chem. Phys., 7, 1103 (1939).

2. M. Avrami, J. Chem. Phys., 8, 212 (1940).

3. M. Avrami, J. Chem. Phys., 9, 177 (1941).

4. K. Nakamura, K. Katayama, and T. Amano, J. Appl. Polym. Sci., 17, 1031 (1973).

5. T. Ozawa, Polymer, 12, 150 (1971).

6. M. Lambrigger, Polym. Eng. Sci. in press.

7. M. Lambrigger, Polym. Eng. Sci., 36, 98 (1996).

8. M. Lambrigger, Polym. J., 29, 188 (1997).

9. T. W. Chan and A. I. Isayev, Polym. Eng. Sci., 34, 461 (1994).

10. W. Kozlowski, J. Polym. Sci., Part C, 38, 47 (1972).

11. T. Sun, J. Pereira, and R. S. Porter, J. Polym. Sci., Polym. Phys. Ed., 22, 1163 (1984).

12. S. A. Jabarin, J. Appl. Polym. Sci., 34, 85 (1987).

13. S. A. Jabarin, J. Appl. Polym. Sci., 34, 97 (1987).

14. C. W. Price, Acta Metall. Mater., 38, 727 (1990). 